\title{
Different QRS morphologies in a dual-chamber pacemaker: what is the mechanism?
}

\author{
Oswaldo Gutiérrez \\ Cardiology Service, Hospital Clínica Bíblica, San José, Costa Rica \\ Correspondence to: Oswaldo Gutiérrez, MD. Hospital Clínica Bíblica, Edificio Strachan, Servicio de Cardiología, Oficina 7, PO Box 471-1300, San \\ José, Costa Rica. Email: oswcr@yahoo.com.ar.
}

\begin{abstract}
A 12-lead electrocardiogram (ECG) of a dual-chamber pacemaker with different paced QRS morphologies is presented. Such an observation is usually made when there are different degrees of fusion, in association with the intrinsic rhythm in the presence of spontaneous changes of the stimulation mode of the pacemaker.
\end{abstract}

Keywords: Pacemaker; stimulation mode; fusion; QRS morphology

Submitted Sep 24, 2017. Accepted for publication Oct 10, 2017.

doi: $10.21037 /$ jtd.2017.10.90

View this article at: http://dx.doi.org/10.21037/jtd.2017.10.90

\section{Clinical vignette}

A dual-chamber pacemaker was implanted in a hypertensive 69 years old male with symptomatic sinus bradycardia. He had a normal ejection fraction and mild concentric left ventricle hypertrophy. A 12-lead electrocardiogram (ECG) was recorded during a routine clinic visit following the implant. Multiple QRS morphologies observed in association with pacemaker stimulation (Figure 1). A major issue and question is what is the mechanism involved?

\section{Discussion of ECG}

The patient's 12-lead ECG is shown with a lead II rhythm strip at the bottom of the tracing. The first beat is stimulated by the pacemaker in DDD mode with an atrial spike, resulting in a $\mathrm{P}$ wave, followed by a ventricular spike, and then a subsequent stimulated broad QRS complex (i.e., atrial stimulation-ventricular stimulation); the next 5 beats are stimulated in a different DDD mode known as the VAT mode, which represents atrial detection-ventricular stimulation. It should be noted that the $\mathrm{P}$ wave is intrinsic and is not preceded by a spike. The different QRS morphology is presumed to be a consequence of a different degree of fusion with the intrinsic ventricular rhythm, which is usually observed when the stimulation mode changes spontaneously. A premature ventricular contraction (PVC), noted in the center of Figure 1, resets the pacemaker, followed by 3 beats in DDD mode, which have the same QRS morphology of the first stimulated QRS. After the PVC, the third beat shows atrial pseudofusion, which consists of an atrial spike delivered at the same time the intrinsic $\mathrm{P}$ wave starts; therefore, its morphology is not changed; this beat and the following beat show fused positive QRS complexes, that suggests predominance of the intrinsic QRS complexes; subsequently VAT mode; the last beat is another PVC, with a morphology differing from the first one.

\section{Points to ponder}

* Different QRS morphologies are frequently observed during normal pacemaker function (1).

* The most common mechanism of different QRS morphologies during a paced rhythm is a different degree of fusion with the intrinsic ventricular rhythm $(2,3)$.

- This condition is usually observed when a spontaneous change occurs in the mode of stimulation or in the intrinsic rhythm of the patient. 


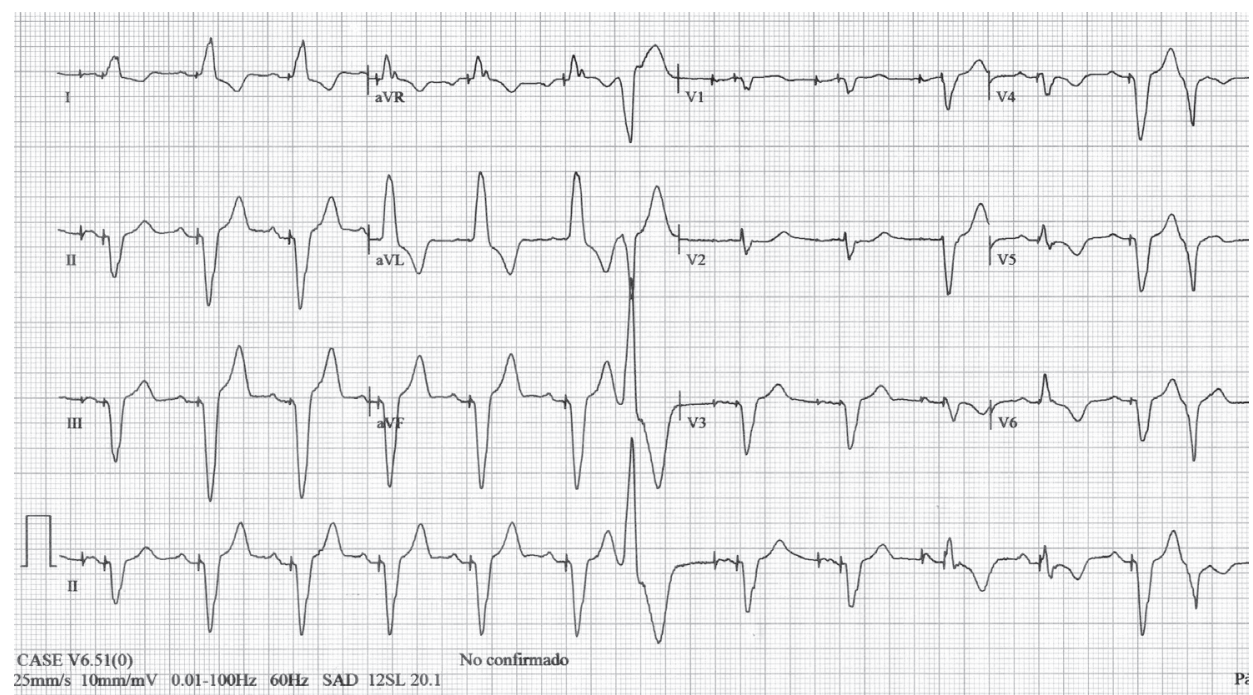

Figure 1 Paced 12-lead electrocardiogram with multiple QRS morphologies.

\section{Acknowledgements}

None.

\section{Footnote}

Conflicts of Interest: The author has no conflicts of interest to declare.

\section{References}

1. Mulpuru SK, Madhavan M, McLeod CJ, et al.

Cite this article as: Gutiérrez O. Different QRS morphologies in a dual-chamber pacemaker: what is the mechanism? J Thorac Dis 2017;9(11):4674-4675. doi: 10.21037/jtd.2017.10.90
Cardiac Pacemakers: Function, Troubleshooting, and Management: Part 1 of a 2-Part Series. J Am Coll Cardiol 2017;69:189-210.

2. Lloyd MS, El Chami MF, Langberg JJ. Pacing features that mimic malfunction: a review of current programmable and automated device functions that cause confusion in the clinical setting. J Cardiovasc Electrophysiol 2009;20:453-60.

3. El-Chami M, Yoo D, Hoskins MH. Intermittent variation in paced QRS morphology: what is the mechanism? Pacing Clin Electrophysiol 2010;33:1267-9. 